\title{
Méthode d'estimation de la prévalence et de l'incidence des handicaps d'origine neurologique / Vaud
}

A.Delachaux, B.Junod, P.-A.Scheder

Institut universitaire de médecine sociale et préventive

Bugnon 17, 1011 Lausanne

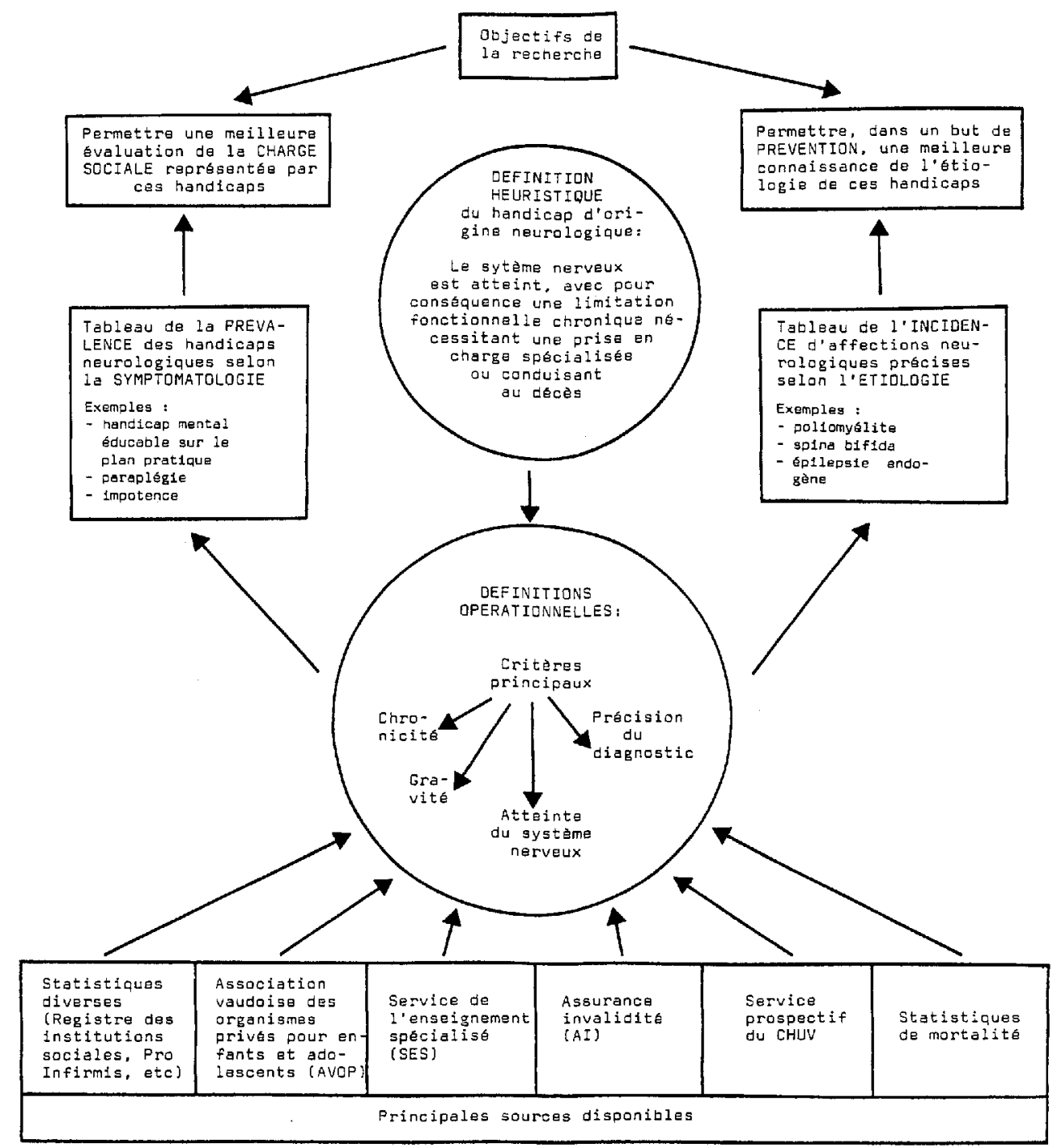

Des chiffres précis concernant la prévalence et l'incidence des handicaps d'origine neuralogique oont nécessajres tant pour ́rvaluer les possibilites de la prévention que pour plenifier la politiqua sociale ingtitutionnalia.

Le Canton de Vaud ne posade pas de registre des handicapés; o'est pourquoi nous avons do rechercher les statistiques flabores par los ingtitutions soclales concernés.

Ces statistiques regroupent des informations conformes eux besolns internes propreg a chaque ingtitution. Elles pressentent des donnés relatives solt à chaque individu (AI, Sarvice proapectif du CHUV) ou institution (Registre des inatitutions sociales, Avap), goit des tableaux synthótiques (SES).

Si certaines sources ne ravetent pour nous qu'une valaur informative, d'autres fournissent des données suffigamment précises pour que nous puiseiong las utiliser lars d'une analyse secondaire (AI, SES, Servicu prospectif du CHUV, statistiques do mortalita).

Notre traveil conaiatera donc a isoler les hendicaper d'origine neurologique permi l'ensemble des ces préantés par les statistiques retenues. C'est pourquai naus alaborarans uno sárle de criteres (dapinitions operationnelias), qui pouvant variar selon les sources cansidarés, permattant d'épurar ces donnés en fanction do notra but. Las criteres aingi retanus concernent principalement:

- la partie atteinte qui doit Btre, entre autros, le système

- la gravitá du handicap,

- sa chronicitá.

Dans las cas où las dannées no sant pas suffisantes pour l'application de ces critures, II s'agira d obtenir des précisions complementalies notamment sur la diagnostic mádical. Sur la base de la symptomatologie, nous obtiendrons alora un indicateur de la prévalence de cos handicaps.

L'átude de l'incidenca devra affiner les statistiques alnal obtenues on effectuant une recherche plus approfondie sur deux cohortes d'enfants, ńs an 1967 at 1971 far exsmplo, pous deux cohortes dienpants, nos an 1967 at 1971 pax axamplo, pour

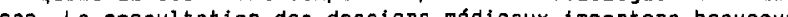
cap. La consultation des dosolars madcaux lmportera baaucoup dans cette ́́tapa. D'ores ot deja nous pouvorie fvaluer à $B D$ le nombre de cas par coharte. 